\title{
Exposita notes
}

\section{Characterizing stochastically monotone functions by multiattribute utility theory}

\author{
Peter Wakker* \\ University of Nijmegen (NICI), P.O. Box 9104, 6500 HE Nijmegen, THE NETHERLANDS
}

Received: September 22, 1991; revised version December 1, 1991

This note shows that the stochastic monotonicity condition, introduced by McKelvey and Page (1986) in the study of common knowledge, is equivalent to a well-known condition from multiattribute utility theory, used before in Harsanyi (1955), Anscombe and Aumann (1963), Fishburn (1965), Keeney and Raiffa (1976), and other places. This also shows how the many generalizations, obtained in the literature on multiattribute utility, can be introduced in the study of common knowledge.

For a concise description of the result from common knowledge, suppose that $n$ individuals observe the value of some stochastically monotone (defined below) aggregate of their individual opinions (e.g., a price). Then this event can only be common knowledge if all individuals assign the same (subjective) expected value to the aggregate. A first version of such a result had been obtained in McKelvey and Page (1987); it was generalized in Theorem 3 of Nielsen et al. (1990). For details the reader is referred to the latter paper. Nielsen et al. obtained their result through an additive representation theorem that was obtained by Bergin and Brandenburger (1990), and that is presented below.

A function $f: \mathbb{R}^{n} \rightarrow \mathbb{R}$ is stochastically monotone if $\int f d \mu>\int f d v$ whenever for the probability distributions $\mu, v$ on $\mathbb{R}^{n}$ these integrals are defined and finite, and the marginal distributions of $\mu$ all weakly stochastically dominate those of $v$ with at least one dominance strict. Bergin and Brandenburger (1990) showed for $f: \mathbb{R}^{n} \rightarrow \mathbb{R}$ :

(i) stochastic monotonicity $\Leftrightarrow$ (ii) additive decomposability into strictly increasing functions.

The result was obtained independently from the literature on multiattribute utility; the latter is explicated for instance in Keeney and Raiffa (1976). This note shows that the result can be obtained as a corollary of multiattribute utility.

The reversed implication (ii) $\Rightarrow$ (i) being elementary, we suppose (i) holds, and derive (ii). In the proof only simple probability distributions will be used, so, without further mention, all distributions below will be assumed to be simple. Write $\mu \geqslant v$

* The research of Peter Wakker has been made possible by a fellowship of the Royal Netherlands Academy of Arts and Sciences. 
whenever $\mu, v$ are probability distributions over $\mathbb{R}^{n}$ with $\int f d \mu \geqq \int f d v$, and $\mu \sim v$ whenever $\int f d \mu=\int f d v$. We say $\geqslant$ satisfies HPc' (a slight weakening of Harsanyi's Postulate $c$ ) if $\mu \sim v$ whenever $\mu, v$ have identical (rather than equivalent, as in Harsanyi, 1955) marginal distributions. This is equivalent to Assumption 2 in Anscombe and Aumann (1963). HPc' is somewhat more general than stochastic monotonicity, and can be derived from stochastic monotonicity: By transitivity of the equivalence relation $\sim$, it suffices to consider the case where the marginals $\mu_{i}$ are independent. Suppose one $\mu_{i}$ is nondegenerate (otherwise $\mu=v$ ), say $\mu_{1}$. Let $\mu^{k}$ be a sequence of probability distributions with independent marginals, obtained from $\mu$ by shifting some probability mass from a low element of $\mu_{1}$ 's support to a high one, with that mass tending to zero. By stochastic monotonicity, $\int f d \mu^{k}>\int f d v$ for all $k$. Since the $\mu^{k}$ 's all have the same support as $\mu$, and only differ concerning assigned probabilities, and since, for a fixed finite support, the integral is linear, so obviously continuous, in probability, the limit of $\int f d \mu^{k}$ is $\int f d \mu$. It follows that $\int f d \mu \geqq \int f d v$. Similarly, the inequality $\int f d \mu \leqq \int f d v$ is derived. From the two inequalities, HPc' follows. By Keeney and Raiffa (1976, Theorem 6.4) it now follows that $f$ is additively decomposable. That result was first obtained in Fishburn (1965); the results of Harsanyi (1955), and Anscombe and Aumann (1963), are similar. The essential idea of the proofs of these results can be summarized as follows:

'If a linear function is a function of linear functions, then the linear function is a linear function of the linear functions.

Coulhon and Mongin (1989) give a clarifying presentation. The special form where all coordinate functions are strictly increasing directly follows from substitution of stochastic monotonicity in the general additive form.

In multiattribute utility, the function $f$ is interpreted as a von Neuman Morgenstern (vNM) utility function. In Harsanyi (1955), this vNM utility function is a social welfare function, and $f_{i}: x_{i} \mapsto f\left(0, \ldots, 0, x_{i}, 0, \ldots, 0\right)$ can be taken as vNM utility function of the $i$ th member of the society. Many generalizations of the above results are known, see for instance Fishburn (1982) and the references therein, or von Stengel (1991).

\section{References}

Anscombe, F.J., Aumann, R.J.: A definition of subjective probability. Ann. Math. Stat. 34, 199-205(1963)

Bergin, J., Brandenburger, A.: A simple characterization of stochastically monotone functions. Econometrica 58, 1241-1243(1990)

Coulhon, T., Mongin, P.: Social choice theory in the case of von Neumann-Morgenstern utilities. Soc. Choice Welfare 6, 175-187 (1989)

Fishburn, P.C.: Independence in utility theory with whole product sets. Operat. Res. 13, 28 45 (1965)

Fishburn, P.C.: The foundations of expected utility. Dordrecht: Reidel 1982

Harsanyi, J.C.: Cardinal welfare, individualistic ethies, and interpersonal comparisons of utility. J. Polit. Econ. 63, 309-321 (1955)

Keeney, R.L., Raiffa, H.: Decisions with multiple objectives. New York: Wiley 1976

McKelvey, R., Page, T.: Common knowledge, consensus, and aggregate information. Econometrica 54, 109-127 (1986)

Nielsen, L.T., Brandenburger, A., Geanakoplos, J., McKelvey, R., Page, T.: Common knowledge of an aggregate of expectations. Econometrica 58, 1235-1239 (1990)

von Stengel, B.: Closure properties of independence concepts for continuous utilities. Math. Operat. Res. (1991) forthcoming 